\title{
Common Fixed Point Theorem in Sequentially Compact Intuitionistic Fuzzy Metric Spaces under Implicit Relations
}

\author{
Seema Mehra \\ Department of Mathematics, \\ M.D.University, Rohtak(INDIA)
}

\begin{abstract}
The aim of this paper is to introduce the notion of sequentially compact intuitionistic fuzzy metric spaces and prove a common fixed point theorem for pairs of weakly compatible self mappings in this newly defined space.
\end{abstract}

\section{Mathematics Subject Classification}

$47 \mathrm{H} 10,54 \mathrm{H} 25$

\section{Keywords}

Intuitionistic fuzzy metric space, sequentially compact intuitionistic fuzzy metric space, compatible mappings, weakly compatible mappings, common fixed point

\section{INTRODUCTION}

As a generalization of fuzzy sets introduced by Zadeh [17], Atanassov [2] introduced the concept of intuitionistic fuzzy sets. Recently, using the idea of intuitionistic fuzzy sets, In 2004, Park[12] defined the notion of intuitionistic fuzzy metric space with the help of continuous t-norms and continuous t-conorms. Recently, in 2006, Alaca et al.[1] using the idea of Intuitionistic fuzzy sets, defined the notion of intuitionistic fuzzy metric space with the help of continuous t-norm and continuous t-conorms as a generalization of fuzzy metric space due to Kramosil and Michalek[10] . In 2006, Turkoglu[16] proved Jungck's[6] common fixed point theorem in the setting of intuitionistic fuzzy metric spaces for commuting mappings. Jungck and Rhoades [6] gave more generalized concept weak compatibility than compatibility. Recently, many authors have studied fixed point theory in intuitionistic fuzzy metric spaces ( [1],[12],[14-16]).Recently, Rao, K.P.R., Rao, K.R.K. and Rao, T. Ranga [13] introduced the concept of sequentially compact fuzzy metric space. Using this concept, we introduce the notion of sequentially compact intuitionistic fuzzy metric spaces and prove a common fixed point theorem for pairs of weakly compatible self mappings in this newly defined space.

\section{PRELIMINARIES}

Definition 1[11] : A binary operation * : $[0,1] \times[0,1]$ $\rightarrow[0,1]$ is a continuous t-norm if it satisfies the following conditions:

(1) $*$ is associative and commutative,

(2) $*$ is continuous,

(3) $\mathrm{a} * 1=$ a for all $\mathrm{a} \in[0,1]$,

(4) $\mathrm{a} * \mathrm{~b} \leq \mathrm{c} * \mathrm{~d}$ whenever $\mathrm{a} \leq \mathrm{c}$ and $\mathrm{b} \leq \mathrm{d}$, for each

$\mathrm{a}, \mathrm{b}, \mathrm{c}, \mathrm{d} \in[0,1]$.
Example 1 : Two typical examples of continuous t-norm are $\mathrm{a} * \mathrm{~b}=\mathrm{ab}$ and $\mathrm{a} * \mathrm{~b}=\min (\mathrm{a}, \mathrm{b})$.

Definition 2[11] : A binary operation $\diamond:[0,1] \times[0,1]$ $\rightarrow[0,1]$ is a continuous $t$-conorm if it satisfies the following conditions :

(1) $\diamond$ is associative and commutative,

(2) $\diamond$ is continuous,

(3) $\mathrm{a} \diamond 0=\mathrm{a}$ for all $\mathrm{a} \in[0,1]$,

(4) a $\diamond$ b $\leq \mathrm{c} \diamond$ d whenever $\mathrm{a} \leq \mathrm{c}$ and $\mathrm{b} \leq \mathrm{d}$,

for each $a, b, c, d \in[0,1]$.

Example 2 : Two typical examples of continuous tconorm are $\mathrm{a} \diamond \mathrm{b}=\min (\mathrm{a}+\mathrm{b}, 1)$ and $\mathrm{a} \diamond \mathrm{b}=\max (\mathrm{a}, \mathrm{b})$.

Remark 1. The concept of triangular norms ( $t$-norms) and triangular conforms ( $t$-conorms) are known as the axiomatic skeletons that we use for characterizing fuzzy intersections and unions respectively. These concepts were originally introduced by Menger [11] in his study of statistical metric spaces. Several examples for these concepts were proposed by many authors ([7-10])

Definition 3[1] : A 5-tuple $(\mathrm{X}, \mathrm{M}, \mathrm{N}, *, \diamond)$ is called a intuitionistic fuzzy metric space if $\mathrm{X}$ is an arbitrary (nonempty) set, $*$ is a continuous t-norm, $\diamond$ a continuous t-conorm and $\mathrm{M}, \mathrm{N}$ are fuzzy sets on $\mathrm{X}^{2} \times[0, \infty)$, satisfying the following conditions : for each $\mathrm{x}, \mathrm{y}, \mathrm{z} \in \mathrm{X}$ and $\mathrm{t}, \mathrm{s}>0$,

(i) $\quad \mathrm{M}(\mathrm{x}, \mathrm{y}, \mathrm{t})+\mathrm{N}(\mathrm{x}, \mathrm{y}, \mathrm{t}) \leq 1$,

(ii) $\mathrm{M}(\mathrm{x}, \mathrm{y}, 0)=0$,

(iii) $\quad \mathrm{M}(\mathrm{x}, \mathrm{y}, \mathrm{t})=1$ if and only if $\mathrm{x}=\mathrm{y}$,

(iv) $\quad \mathrm{M}(\mathrm{x}, \mathrm{y}, \mathrm{t})=\mathrm{M}(\mathrm{y}, \mathrm{x}, \mathrm{t})$,

(v) $\quad \mathrm{M}(\mathrm{x}, \mathrm{y}, \mathrm{t}) * \mathrm{M}(\mathrm{y}, \mathrm{z}, \mathrm{s}) \leq \mathrm{M}(\mathrm{x}, \mathrm{z}, \mathrm{t}+\mathrm{s})$,

(vi) $\quad \mathrm{M}(\mathrm{x}, \mathrm{y},):.[0, \infty) \rightarrow[0,1]$ is left continuous, $\lim$

(viii) $\quad \mathrm{N}(\mathrm{x}, \mathrm{y}, 0)=1$,

(ix) $\quad \mathrm{N}(\mathrm{x}, \mathrm{y}, \mathrm{t})=0$ if and only if $\mathrm{x}=\mathrm{y}$,

(x) $\quad \mathrm{N}(\mathrm{x}, \mathrm{y}, \mathrm{t})=\mathrm{N}(\mathrm{y}, \mathrm{x}, \mathrm{t})$,

(xi) $\quad \mathrm{N}(\mathrm{x}, \mathrm{y}, \mathrm{t}) \diamond \mathrm{N}(\mathrm{y}, \mathrm{z}, \mathrm{s}) \geq \mathrm{N}(\mathrm{x}, \mathrm{z}, \mathrm{t}+\mathrm{s})$,

(xii) $\mathrm{N}(\mathrm{x}, \mathrm{y},):.[0, \infty) \rightarrow[0,1]$ is right continuous, 


$$
\lim
$$

$$
t \rightarrow \infty \mathrm{N}(\mathrm{x}, \mathrm{y}, \mathrm{t})=0 .
$$

Then $(\mathrm{M}, \mathrm{N})$ is called an intuitionistic fuzzy metric on $\mathrm{X}$. The functions $M(x, y, t)$ and $N(x, y, t)$ denote the degree of nearness and the degree of non-nearness between $\mathrm{x}$ and $\mathrm{y}$ with respect to t, respectively.

Remark 2 : Every fuzzy metric space $(\mathrm{X}, \mathrm{M}, *)$ is an intuitionistic fuzzy metric space of the form (X,M, 1-M, *, $\diamond)$ such that $\mathrm{t}$-norm $*$ and $\mathrm{t}$-conorm $\diamond$ are associated [12], i.e. $\mathrm{x} \diamond$ $\mathrm{y}=1-[(1-\mathrm{x}) *(1-\mathrm{y})]$ for any $\mathrm{x}, \mathrm{y} \in \mathrm{X}$.

Remark 3 : In intuitionistic fuzzy metric space X,

$\mathrm{M}(\mathrm{x}, \mathrm{y},$.$) is non-decreasing and \mathrm{N}(\mathrm{x}, \mathrm{y},$.$) is non-increasing$ for all

$\mathrm{x}, \mathrm{y} \in \mathrm{X}$.

Example 3 : (Induced intuitionistic fuzzy metric).

Let $(\mathrm{X}, \mathrm{d})$ be a metric space. Denote $\mathrm{a} * \mathrm{~b}=\mathrm{ab}$ and $\mathrm{a} \diamond \mathrm{b}=$ $\min (a+b, 1)$ for all $a, b \in[0,1]$ and let $M_{d}$ and $N_{d}$ be fuzzy sets on $\mathrm{X}^{2} \times[0, \infty)$ defined as follows:

$$
\begin{aligned}
& \mathrm{M}_{\mathrm{d}}(\mathrm{x}, \mathrm{y}, \mathrm{t})=\frac{h t^{n}}{h t^{n}+m d(x, y)} \\
& \mathrm{N}_{\mathrm{d}}(\mathrm{x}, \mathrm{y}, \mathrm{t})=\frac{d(x, y)}{k t^{n}+m d(x, y)}
\end{aligned}
$$

for all $h, k, m, n \in R^{+}$. Then $\left(X, M_{d}, N_{d}, *, \diamond\right)$ is an intuitionistic fuzzy metric space.

Definition 4 : Let $(\mathrm{X}, \mathrm{M}, \mathrm{N}, *, \diamond)$ be an intuitionistic fuzzy metric space. Then

(a) a sequence $\left\{x_{n}\right\}$ in $X$ is said to be Cauchy sequence if, for all $\mathrm{t}>0$ and $\mathrm{p}>0$,

$$
\lim _{n \rightarrow \infty} \mathrm{M}\left(\mathrm{x}_{\mathrm{n}+\mathrm{p}}, \mathrm{x}_{\mathrm{n}}, \mathrm{t}\right)=1, \lim _{n \rightarrow \infty} \mathrm{N}\left(\mathrm{x}_{\mathrm{n}+\mathrm{p}}, \mathrm{x}_{\mathrm{n}}, \mathrm{t}\right)=0 .
$$

(b) a sequence $\left\{x_{n}\right\}$ in $X$ is said to be convergent to a point $\mathrm{x}$ $\in \mathrm{X}$ if, for all $\mathrm{t}>0$

$$
\lim _{n \rightarrow \infty} \mathrm{M}\left(\mathrm{x}_{\mathrm{n}}, \mathrm{x}, \mathrm{t}\right)=1 \text { and } \lim _{n \rightarrow \infty} \mathrm{N}\left(\mathrm{x}_{\mathrm{n}}, \mathrm{x}, \mathrm{t}\right)=0 .
$$

Since $*$ and $\diamond$ are continuous, the limit is uniquely determined from (v) and (xi), respectively.

Definition 5: An intuitionistic fuzzy metric space $(\mathrm{X}, \mathrm{M}, \mathrm{N}, *, \diamond)$ is said to be complete if every Cauchy sequence is convergent.

Definition 6 : Let $\mathrm{A}$ and $\mathrm{B}$ be mappings from an intuitionistic fuzzy metric space $(X, M, N, *, \diamond)$ in to itself. The mappings $\mathrm{A}$ and $\mathrm{B}$ are said to be compatible if

$$
\lim _{n \rightarrow \infty} \mathrm{M}\left(\mathrm{ABx}_{\mathrm{n}}, \mathrm{BAx}_{\mathrm{n}}, \mathrm{t}\right)=1 \text {, }
$$

$$
\lim _{n \rightarrow \infty} \mathrm{N}\left(\mathrm{ABx}_{\mathrm{n}}, \mathrm{BAx}_{\mathrm{n}}, \mathrm{t}\right)=0
$$

for all $t>0$, whenever $\left\{x_{n}\right\}$ is a sequence in $X$ such that

$$
\lim _{n \rightarrow \infty} \mathrm{Ax}_{\mathrm{n}}=\lim _{n \rightarrow \infty} \mathrm{Bx}_{\mathrm{n}}=\mathrm{z} \text { for some } \mathrm{z} \in \mathrm{X}
$$

Definition 7 : Self mappings A and B of an intuitionistic fuzzy metric space $(\mathrm{X}, \mathrm{M}, \mathrm{N}, *, \diamond)$ is said to be weakly compatible if they commute at their coincidence point, that is,

$\mathrm{Ax}=\mathrm{Bx}$ implies that $\mathrm{ABx}=\mathrm{BAx}$ for some $\mathrm{x} \in \mathrm{X}$.

It is easy to see that if self mappings $\mathrm{A}$ and $\mathrm{B}$ of an intuitionistic fuzzy metric space $(\mathrm{X}, \mathrm{M}, \mathrm{N}, *, \diamond)$ is compatible then they are weakly compatible.

The following example shows that the converse of above statement does

not hold.

Example 4 : Let $(X, M, N, *, \diamond)$ be an intuitionistic fuzzy metric space, Where $X=[0,2]$ with t-norm and t-conorm defined by $\mathrm{a} * \mathrm{~b}=\min \{\mathrm{a}, \mathrm{b}\}$ and $\mathrm{a} \diamond \mathrm{b}=\max \{\mathrm{a}, \mathrm{b}\}$, for all $\mathrm{a}$, $\mathrm{b} \in[0,1]$ and

$\mathrm{M}_{\mathrm{d}}(\mathrm{x}, \mathrm{y}, \mathrm{t})=\frac{t}{t+d(x, y)}, \mathrm{N}_{\mathrm{d}}(\mathrm{x}, \mathrm{y}, \mathrm{t})=\frac{d(x, y)}{t+d(x, y)}$ for

all $\mathrm{t}>0$

and $\mathrm{M}_{d}(\mathrm{x}, \mathrm{y}, \mathrm{t})=0$ and $\mathrm{N}_{\mathrm{d}}(\mathrm{x}, \mathrm{y}, \mathrm{t})=1$ for $\mathrm{t}=0$, for all $\mathrm{x}, \mathrm{y} \in$ $\mathrm{X}$.

Define self maps A and B on X as follows:

$\mathrm{Ax}=\left\{\begin{array}{lr}2 & \text { if } 0 \leq x \leq 1 \\ \frac{x}{2} & \text { if } 1<x \leq 2\end{array}\right.$

$\mathrm{Bx}=\left\{\begin{aligned} 2 & \text { if } x=1 \\ \frac{x+3}{5} & \text { otherwise }\end{aligned}\right.$

And $x_{n}=2-\frac{1}{(2 n)}$. Then we have

$\mathrm{A}(1)=\mathrm{B}(1)=2$ and $\mathrm{A}(2)=\mathrm{B}(2)=1$

Also $\mathrm{AB}(1)=\mathrm{BA}(1)=2$. Thus $(\mathrm{A}, \mathrm{B})$ is weak compatible.Again

$\mathrm{Ax}_{\mathrm{n}}=1-\frac{1}{4 n}, \mathrm{Bx}_{\mathrm{n}}=1-\frac{1}{10 n}$

Thus $\mathrm{Ax}_{\mathrm{n}}=1, \mathrm{Bx}_{\mathrm{n}}=1$

Hence $\mathrm{z}=1$.

Further $\mathrm{ABx}_{\mathrm{n}}=2, \mathrm{BAx}_{\mathrm{n}}=\frac{4}{5}-\frac{1}{20 n}$ 


$$
\begin{aligned}
& \text { Now } \lim _{n \rightarrow \infty} \mathrm{M}\left(\mathrm{ABx}_{\mathrm{n}}, \mathrm{BAx}_{\mathrm{n}}, \mathrm{t}\right)=\lim _{\mathrm{n} \rightarrow \infty} \mathrm{M}\left(2, \frac{4}{5}-\frac{1}{20 n},\right. \\
& \mathrm{t}=\frac{t}{t+\frac{6}{5}} \neq 1,
\end{aligned}
$$$$
\lim _{n \rightarrow \infty} \mathrm{N}\left(\mathrm{ABX}_{\mathrm{n}}, \mathrm{BAx_{n }}, \mathrm{t}\right)=\lim _{\mathrm{n} \rightarrow \infty} \mathrm{N}\left(2, \frac{4}{5}-\frac{1}{20 n}, \mathrm{t}\right)=
$$$$
\frac{\frac{6}{5}}{t+\frac{6}{5}} \neq 0, \mathrm{t}>0
$$

Hence (A, B) is not compatible.

Definition 8. $(X, M, N, *, \diamond)$ is said to be sequentially compact intuitionistic fuzzy metric space if every sequence in $\mathrm{X}$ has a convergent sub sequence in it.

Let $\Phi$ be the set of all functions $\phi, \phi^{\prime}:[0,1]^{5} \rightarrow[0,1]$ such that

(i) $\phi, \phi^{\prime}$ are non decreasing and non increasing in all coordinates respectively,

(ii) $\phi\left(\mathrm{t}_{1}, \mathrm{t}_{2}, \mathrm{t}_{3}, \mathrm{t}_{4}, \mathrm{t}_{5}\right), \phi^{\prime}\left(\mathrm{t}_{1}, \mathrm{t}_{2}, \mathrm{t}_{3}, \mathrm{t}_{4}, \mathrm{t}_{5}\right)$ are continuous in $\mathrm{t}_{4}$ and $t_{5}$ and

(iii) $\phi(t, t, t, t, t)>t, \phi^{\prime}(t, t, t, t, t)<t$ for every $t \in[0,1)$.

All material on each page should fit within a rectangle of $18 \mathrm{x}$ $23.5 \mathrm{~cm}$ (7" x 9.25"), centered on the page, beginning $2.54 \mathrm{~cm}$ (1") from the top of the page and ending with $2.54 \mathrm{~cm}(1 ")$ from the bottom. The right and left margins should be $1.9 \mathrm{~cm}$ (.75"). The text should be in two $8.45 \mathrm{~cm}(3.33 ")$ columns with a $.83 \mathrm{~cm}(.33 ")$ gutter.

\section{MAIN RESULTS}

Here afterwards, assume that $\left(\mathrm{X}, \mathrm{M}, \mathrm{N},{ }^{*}, \diamond\right)$ be a sequentially compact intuitionistic fuzzy metric space with $\mathrm{t} * \mathrm{t} \geq \mathrm{t}, \mathrm{s} \diamond \mathrm{s}$ $\leq \mathrm{s} \forall \mathrm{t}, \mathrm{s} \in[0,1]$.

Theorem 3.1 Let P, Q, A, B, Sand T be self-mappings on $(\mathrm{X}, \mathrm{M}, \mathrm{N}, *, \diamond)$ such that

(i) $\mathrm{P}(\mathrm{X}) \subset \mathrm{ST}(\mathrm{X})$ and $\mathrm{Q}(\mathrm{X}) \subset \mathrm{AB}(\mathrm{X})$,

(ii) $\mathrm{P}$ and $\mathrm{AB}$ are continuous or $\mathrm{Q}$ and $\mathrm{ST}$ are continuous,

(iii) $\mathrm{AB}=\mathrm{BA}, \mathrm{ST}=\mathrm{TS}, \mathrm{PB}=\mathrm{BP}, \mathrm{TQ}=\mathrm{QT}$,

(iv) the pairs $(\mathrm{P}, \mathrm{AB})$ and $(\mathrm{Q}, \mathrm{ST})$ are weakly compatible,

(v) $[1+\mathrm{aM}(\mathrm{ABx}, \mathrm{STy}, \mathrm{t})] * \mathrm{M}(\mathrm{Px}, \mathrm{Qy}, \mathrm{t})$

$$
\geq \mathrm{a}[\mathrm{M}(\mathrm{ABx}, \mathrm{STy}, \mathrm{t}) * \mathrm{M}(\mathrm{Px}, \mathrm{ABx}, \mathrm{t}) * \mathrm{M}(\mathrm{Qy}, \mathrm{STy}, \mathrm{t})
$$

* M(Px,STy, $\alpha \mathrm{t}) * \mathrm{M}(\mathrm{Qy}, \mathrm{ABx},(2-\alpha) \mathrm{t})]+$

$$
\begin{gathered}
\phi\left(\begin{array}{c}
M(A B x, S T y, t), M(P x, A B x, t), M(Q y, S T y, t) \\
M(P x, S T y, \alpha t), M(Q y, A B x,(2-\alpha) t)
\end{array}\right) \\
{[1+\mathrm{aN}(\mathrm{ABx}, \mathrm{STy}, \mathrm{t})] \diamond \mathrm{N}(\mathrm{Px}, \mathrm{Qy}, \mathrm{t})} \\
\leq \mathrm{a}[\mathrm{N}(\mathrm{ABx}, \mathrm{STy}, \mathrm{t}) \diamond \mathrm{N}(\mathrm{Px}, \mathrm{ABx}, \mathrm{t}) \diamond \mathrm{N}(\mathrm{Qy}, \mathrm{STy}, \mathrm{t}) \\
\diamond \mathrm{N}(\mathrm{Px}, \mathrm{STy}, \alpha \mathrm{t}) \diamond \mathrm{N}(\mathrm{Qy}, \mathrm{ABx},(2-\alpha) \mathrm{t})]+ \\
\phi^{\prime}\left(\begin{array}{c}
N(A B x, S T y, t), N(P x, A B x, t), N(Q y, S T y, t) \\
N(P x, S T y, \alpha t), N(Q y, A B x,(2-\alpha) t)
\end{array}\right)
\end{gathered}
$$

for every $\mathrm{x}, \mathrm{y} \in \mathrm{X}$, for all $\mathrm{t}>0$ and for every $\alpha \in(0,2)$, where $\phi, \phi^{\prime} \in \Phi$ and $\mathrm{a} \in \mathbf{R}$, Then $\mathrm{P}, \mathrm{Q}, \mathrm{A}, \mathrm{B}, \mathrm{S}$ and $\mathrm{T}$ have a unique common fixed point $\mathrm{p}$ in $\mathrm{X}$.

Proof. Suppose $\mathrm{P}$ and $\mathrm{AB}$ are continuous.

For every $\mathrm{t}>0$, let $\mathrm{m}=\sup \{\mathrm{M}(\mathrm{Px}, \mathrm{ABx}, \mathrm{t}): \mathrm{x} \in \mathrm{X}\}$,

$$
\text { and } \mathrm{n}=\inf \{\mathrm{N}(\mathrm{Px}, \mathrm{ABx}, \mathrm{t}): \mathrm{x} \in \mathrm{X}\} \text {, }
$$

Since $\mathrm{P}$ and $\mathrm{AB}$ are continuous on sequentially compact intuitionistic fuzzy metric space, there exists $\mathrm{u} \in \mathrm{X}$ such that $\mathrm{m}=\mathrm{M}(\mathrm{Pu}, \mathrm{ABu}, \mathrm{t}), \mathrm{n}=\mathrm{N}(\mathrm{Pu}, \mathrm{ABu}, \mathrm{t})$

Since $\mathrm{P}(X) \subset \mathrm{ST}(\mathrm{X})$, there exists $\mathrm{v} \in \mathrm{X}$ such that $\mathrm{Pu}=$ STv...... (vi)

Suppose $\mathrm{m}<1$ and $\mathrm{n} \geq 0$.

Putting $\mathrm{x}=\mathrm{u}, \mathrm{y}=\mathrm{v}, \alpha=1-\mathrm{q}_{1}, \mathrm{q}_{1} \in(0,1)$ in (v) we have

$[1+\mathrm{aM}(\mathrm{ABu}, \mathrm{STv}, \mathrm{t})] * \mathrm{M}(\mathrm{Pu}, \mathrm{Qv}, \mathrm{t})$

$\geq \mathrm{a}[\mathrm{M}(\mathrm{ABu}, \mathrm{STv}, \mathrm{t}) * \mathrm{M}(\mathrm{Pu}, \mathrm{ABu}, \mathrm{t}) * \mathrm{M}(\mathrm{Qv}, \mathrm{STv}, \mathrm{t}) *$

$\left.\mathrm{M}\left(\mathrm{Pu}, \mathrm{STv},\left(1-\mathrm{q}_{1}\right) \mathrm{t}\right) * \mathrm{M}\left(\mathrm{Qv}, \mathrm{ABu},\left(1+\mathrm{q}_{1}\right) \mathrm{t}\right)\right]+$

$\phi\left(\begin{array}{c}M(A B u, S T v, t), M(P u, A B u, t), M(Q v, S T v, t) \\ M\left(P u, S T v,\left(1-q_{1}\right) t\right), M\left(Q v, A B u,\left(1+q_{1}\right) t\right)\end{array}\right)$

$[1+\mathrm{aN}(\mathrm{ABu}, \mathrm{STv}, \mathrm{t})] \diamond \mathrm{N}(\mathrm{Pu}, \mathrm{Qv}, \mathrm{t})$

$\leq \mathrm{a}[\mathrm{N}(\mathrm{ABu}, \mathrm{STv}, \mathrm{t}) \diamond \mathrm{N}(\mathrm{Pu}, \mathrm{ABu}, \mathrm{t}) \diamond \mathrm{N}(\mathrm{Qv}, \mathrm{ST} \mathrm{v}, \mathrm{t}) \diamond$

$\left.\mathrm{N}\left(\mathrm{Pu}, \mathrm{STv},\left(1-\mathrm{q}_{1}\right) \mathrm{t}\right) \diamond \mathrm{N}\left(\mathrm{Qv}, \mathrm{ABu},\left(1+\mathrm{q}_{1}\right) \mathrm{t}\right)\right]+$

$\phi^{\prime}\left(\begin{array}{c}N(A B u, S T v, t), N(P u, A B u, t), N(Q v, S T v, t) \\ N\left(P u, S T v,\left(1-q_{1}\right) t\right), N\left(Q v, A B u,\left(1+q_{1}\right) t\right)\end{array}\right)$

$[1+\mathrm{am}] * \mathrm{M}(\mathrm{STv}, \mathrm{Qv}, \mathrm{t}) \geq \mathrm{a}[\mathrm{m} * \mathrm{~m} * \mathrm{M}(\mathrm{Qv}, \mathrm{STv}, \mathrm{t}) * 1 * \mathrm{~m}$

$\left.* \mathrm{M}\left(\mathrm{Qv}, \mathrm{STv}, \mathrm{q}_{1} \mathrm{t}\right)\right]+\phi(\mathrm{m}, \mathrm{m}, \mathrm{M}(\mathrm{Qv}, \mathrm{STv}, \mathrm{t}), 1, \mathrm{~m} * \mathrm{M}(\mathrm{Qv}$,

STv, $\left.\mathrm{q}_{1} \mathrm{t}\right)$ ), 
$[1+\mathrm{a} n] \diamond \mathrm{N}(\mathrm{STv}, \mathrm{Qv}, \mathrm{t}) \leq \mathrm{a}\left[\mathrm{n}{ }_{\mathrm{n}} \diamond_{\mathrm{N}}(\mathrm{Qv}, \mathrm{STv}, \mathrm{t}) \diamond_{0} \diamond_{\mathrm{n}}\right.$ $\left.\diamond \mathrm{N}\left(\mathrm{Qv}, \mathrm{STv}, \mathrm{q}_{1} \mathrm{t}\right)\right]+\phi^{\prime}(\mathrm{n}, \mathrm{n}, \mathrm{N}(\mathrm{Qv}, \mathrm{STv}, \mathrm{t}), 0, \mathrm{n} \oslash \mathrm{N}(\mathrm{Qv}$,

STv, $\left.\left.\mathrm{q}_{1} \mathrm{t}\right)\right)$

Letting $\mathrm{q}_{1} \rightarrow 1$, we have

$[1+\mathrm{am}] * \mathrm{M}(\mathrm{STv}, \mathrm{Qv}, \mathrm{t}) \geq \mathrm{a}[\mathrm{m} * \mathrm{M}(\mathrm{STv}, \mathrm{Qv}, \mathrm{t})]+$

$$
\phi(\mathrm{m}, \mathrm{m}, \mathrm{M}(\mathrm{Qv}, \mathrm{STv}, \mathrm{t}), 1, \mathrm{M}(\mathrm{Qv}, \mathrm{STv}, \mathrm{t}) * \mathrm{~m}),
$$

$[1+\mathrm{an}] \diamond \mathrm{N}(\mathrm{ST}, \mathrm{Qv}, \mathrm{t}) \leq \mathrm{a}\left[\mathrm{n} \diamond \mathrm{n} \diamond \mathrm{N}(\mathrm{Qv}, \mathrm{STv}, \mathrm{t}) \diamond_{0} \diamond \mathrm{n}\right.$ $\left.\diamond \mathrm{N}\left(\mathrm{Qv}, \mathrm{STv}, \mathrm{q}_{1} \mathrm{t}\right)\right]+\phi^{\prime}(\mathrm{n}, \mathrm{n}, \mathrm{N}(\mathrm{Qv}, \mathrm{STv}, \mathrm{t}), 0, \mathrm{n} \oslash \mathrm{N}(\mathrm{Qv}$, STv, $\left.\left.\mathrm{q}_{1} \mathrm{t}\right)\right)$

$\mathrm{M}(\mathrm{STv}, \mathrm{Qv}, \mathrm{t}) \geq \varphi(\mathrm{m}, \mathrm{m}, \mathrm{M}(\mathrm{Qv}, \mathrm{STv}, \mathrm{t}), 1, \mathrm{M}(\mathrm{Qv}, \mathrm{STv}, \mathrm{t}) * \mathrm{~m})$, $\mathrm{N}(\mathrm{STv}, \mathrm{Qv}, \mathrm{t}) \leq \phi^{\prime}(\mathrm{n}, \mathrm{n}, \mathrm{N}(\mathrm{Qv}, \mathrm{STv}, \mathrm{t}), 0, \mathrm{~N}(\mathrm{Qv}, \mathrm{STv}, \mathrm{t}) \diamond$ n),

If $\mathrm{m} \geq \mathrm{M}(\mathrm{Qv}, \mathrm{STv}, \mathrm{t})=\mathrm{r}$ and $\mathrm{n} \leq \mathrm{N}(\mathrm{Qv}, \mathrm{STv}, \mathrm{t})=\mathrm{r}$ then using $\mathrm{t} * \mathrm{t} \geq \mathrm{t}, \mathrm{s} \nabla_{\mathrm{s}} \leq \mathrm{s}$, we have

$\mathrm{r}=\mathrm{M}(\mathrm{Qv}, \mathrm{STv}, \mathrm{t}) \geq \phi(\mathrm{r}, \mathrm{r}, \mathrm{r}, \mathrm{r}, \mathrm{r})>\mathrm{r}, \mathrm{r}=\mathrm{N}(\mathrm{Qv}, \mathrm{STv}, \mathrm{t}) \leq$ $\phi^{\prime}(\mathrm{r}, \mathrm{r}, \mathrm{r}, \mathrm{r}, \mathrm{r})<\mathrm{r}$.

It is a contradiction. Hence we have

$\mathrm{m}<\mathrm{M}(\mathrm{Qv}, \mathrm{STv}, \mathrm{t})$ and $\mathrm{n}>\mathrm{N}(\mathrm{Qv}, \mathrm{STv}, \mathrm{t})$

Since $\mathrm{Q}(\mathrm{X}) \subseteq \mathrm{AB}(\mathrm{X})$, there exists $\mathrm{w} \in \mathrm{X}$ such that $\mathrm{Qv}=$ $\mathrm{ABw}$......(viii)

Now $\mathrm{M}(\mathrm{Pw}, \mathrm{ABw}, \mathrm{t}) \leq \mathrm{m}<1$ and $\mathrm{N}(\mathrm{Pw}, \mathrm{ABw}, \mathrm{t})>\mathrm{n} \geq 0$. From (ii), with $\alpha=1+\mathrm{q}_{2}$,

$\mathrm{q}_{2} \in(0,1)$ we have

$[1+\mathrm{aM}(\mathrm{ABw}, \mathrm{STv}, \mathrm{t})] * \mathrm{M}(\mathrm{Pw}, \mathrm{Qv}, \mathrm{t})$

$\geq \mathrm{a}[\mathrm{M}(\mathrm{ABw}, \mathrm{STv}, \mathrm{t}) * \mathrm{M}(\mathrm{Pw}, \mathrm{ABw}, \mathrm{t}) * \mathrm{M}(\mathrm{Qv}, \mathrm{STv}, \mathrm{t}) *$

$\left.\mathrm{M}\left(\mathrm{Pw}, \mathrm{STv},\left(1+\mathrm{q}_{2}\right) \mathrm{t}\right) * \mathrm{M}\left(\mathrm{Qv}, \mathrm{ABw},\left(1-\mathrm{q}_{2}\right) \mathrm{t}\right)\right]+$

$\phi\left(\begin{array}{c}M(A B w, S T v, t), M(P w, A B w, t), M(Q v, S T v, t) \\ M\left(P w, S T v,\left(1+q_{2}\right) t\right), M\left(Q v, A B w,\left(1-q_{2}\right) t\right)\end{array}\right)$

$[1+\mathrm{aN}(\mathrm{ABw}, \mathrm{STv}, \mathrm{t})] \diamond \mathrm{N}(\mathrm{Pw}, \mathrm{Qv}, \mathrm{t})$

$\leq \mathrm{a}[\mathrm{N}(\mathrm{ABw}, \mathrm{STv}, \mathrm{t}) \diamond \mathrm{N}(\mathrm{Pw}, \mathrm{ABw}, \mathrm{t}) \diamond \mathrm{N}(\mathrm{Qv}, \mathrm{STv}, \mathrm{t}) \diamond$ $\left.\mathrm{N}\left(\mathrm{Pw}, \mathrm{STv},\left(1+\mathrm{q}_{2}\right) \mathrm{t}\right) \diamond \mathrm{N}\left(\mathrm{Qv}, \mathrm{ABw},\left(1-\mathrm{q}_{2}\right) \mathrm{t}\right)\right]+$

$\phi^{\prime}\left(\begin{array}{c}N(A B w, S T v, t), N(P w, A B w, t), N(Q v, S T v, t) \\ N\left(P w, S T v,\left(1+q_{2}\right) t\right), N\left(Q v, A B w,\left(1-q_{2}\right) t\right)\end{array}\right)$
$[1+\mathrm{aM}(\mathrm{Qv}, \mathrm{STv}, \mathrm{t})] * \mathrm{M}(\mathrm{Pw}, \mathrm{ABw}, \mathrm{t})$

$\geq \mathrm{a}[\mathrm{M}(\mathrm{Qv}, \mathrm{STv}, \mathrm{t}) * \mathrm{M}(\mathrm{Pw}, \mathrm{ABw}, \mathrm{t}) * \mathrm{M}(\mathrm{Qv}, \mathrm{STv}, \mathrm{t}) *$ $\left.\mathrm{M}(\mathrm{Pw}, \mathrm{ABw}, \mathrm{t}) * \mathrm{M}\left(\mathrm{Qv}, \mathrm{STv}, \mathrm{q}_{2} \mathrm{t}\right) * 1\right]+$

$\phi\left(\begin{array}{c}M(Q v, S T v, t), M(P w, A B w, t), M(Q v, S T v, t) \\ M(P w, A B w, t) * M\left(Q v, S T v, q_{2} t\right), 1\end{array}\right)$

$[1+\mathrm{aN}(\mathrm{Qv}, \mathrm{STv}, \mathrm{t})] \diamond \mathrm{N}(\mathrm{Pw}, \mathrm{ABw}, \mathrm{t})$

$\leq \mathrm{a}[\mathrm{N}(\mathrm{Qv}, \mathrm{STv}, \mathrm{t}) \diamond \mathrm{N}(\mathrm{Pw}, \mathrm{ABw}, \mathrm{t}) \diamond \mathrm{N}(\mathrm{Qv}, \mathrm{STv}, \mathrm{t}) \diamond$ $\left.\mathrm{N}(\mathrm{Pw}, \mathrm{ABw}, \mathrm{t}) \diamond \mathrm{N}\left(\mathrm{Qv}, \mathrm{STv}, \mathrm{q}_{2} \mathrm{t}\right) \diamond 0\right]+$

$\phi^{\prime}\left(\begin{array}{c}N(Q v, S T v, t), N(P w, A B w, t), N(Q v, S T v, t) \\ N(P w, A B w, t) \diamond N\left(Q v, S T v, q_{2} t\right), 0\end{array}\right)$

Letting $\mathrm{q}_{2} \rightarrow 1$, we have

$[1+\mathrm{aM}(\mathrm{Qv}, \mathrm{STv}, \mathrm{t})] * \mathrm{M}(\mathrm{Pw}, \mathrm{ABw}, \mathrm{t})$

$\geq \mathrm{a}[\mathrm{M}(\mathrm{Qv}, \mathrm{STv}, \mathrm{t})] * \mathrm{M}(\mathrm{Pw}, \mathrm{ABw}, \mathrm{t})+$

$\phi\left(\begin{array}{c}M(Q v, S T v, t), M(P w, A B w, t), M(Q v, S T v, t) \\ M(Q v, S T v, t) * M(P w, A B w, t), 1\end{array}\right)$

$[1+\mathrm{aN}(\mathrm{Qv}, \mathrm{STv}, \mathrm{t})] \diamond \mathrm{N}(\mathrm{Pw}, \mathrm{ABw}, \mathrm{t})$

$\leq \mathrm{a}[\mathrm{N}(\mathrm{Qv}, \mathrm{STv}, \mathrm{t})] \diamond \mathrm{N}(\mathrm{Pw}, \mathrm{ABw}, \mathrm{t})+$

$\phi^{\prime}\left(\begin{array}{c}N(Q v, S T v, t), N(P w, A B w, t), N(Q v, S T v, t) \\ N(Q v, S T v, t) \oslash N(P w, A B w, t), 0\end{array}\right)$

$\mathrm{M}(\mathrm{Pw}, \mathrm{ABw}, \mathrm{t}) \geq$

$\phi\left(\begin{array}{c}M(Q v, S T v, t), M(P w, A B w, t), M(Q v, S T v, t) \\ M(Q v, S T v, t) * M(P w, A B w, t), 1\end{array}\right)$

$\mathrm{N}(\mathrm{Pw}, \mathrm{ABw}, \mathrm{t}) \leq$

$\phi^{\prime}\left(\begin{array}{c}N(Q v, S T v, t), N(P w, A B w, t), N(Q v, S T v, t) \\ N(Q v, S T v, t) \diamond N(P w, A B w, t), 0\end{array}\right)$

If $\mathrm{M}(\mathrm{Qv}, \mathrm{STv}, \mathrm{t}) \geq \mathrm{M}(\mathrm{Pw}, \mathrm{ABw}, \mathrm{t})$

$=\mathrm{s}=\mathrm{N}(\mathrm{Qv}, \mathrm{STv}, \mathrm{t})<\mathrm{N}(\mathrm{Pw}, \mathrm{ABw}, \mathrm{t})$ then 
$\mathrm{M}(\mathrm{Pw}, \mathrm{ABw}, \mathrm{t}) \geq \varphi(\mathrm{s}, \mathrm{s}, \mathrm{s}, \mathrm{s}, \mathrm{s})>\mathrm{s}$ and

$\mathrm{N}(\mathrm{Pw}, \mathrm{ABw}, \mathrm{t}) \leq \phi^{\prime}(\mathrm{s}, \mathrm{s}, \mathrm{s}, \mathrm{s}, \mathrm{s})<\mathrm{s}$

It is a contradiction. Hence we have

$\mathrm{M}(\mathrm{Qv}, \mathrm{STv}, \mathrm{t})<\mathrm{M}(\mathrm{Pw}, \mathrm{ABw}, \mathrm{t})$

$\mathrm{N}(\mathrm{Qv}, \mathrm{STv}, \mathrm{t})>\mathrm{N}(\mathrm{Pw}, \mathrm{ABw}, \mathrm{t})$......(ix).

Now from definition of $\mathrm{m}, \mathrm{n}$ and (ix), (vii) we have

$\mathrm{m} \geq \mathrm{M}(\mathrm{Pw}, \mathrm{ABw}, \mathrm{t})>\mathrm{M}(\mathrm{Qv}, \mathrm{STv}, \mathrm{t})>\mathrm{m}$,

$\mathrm{n} \leq \mathrm{N}(\mathrm{Pw}, \mathrm{ABw}, \mathrm{t})<\mathrm{N}(\mathrm{Qv}, \mathrm{STv}, \mathrm{t})<\mathrm{n}$ It is a contradiction.

Hence $\mathrm{m}=1$ and $\mathrm{n}=0$.

Thus $\mathrm{Pu}=\mathrm{ABu} \ldots \ldots .(\mathrm{x})$

Suppose $\mathrm{M}(\mathrm{Qv}, \mathrm{STv}, \mathrm{t})<1, \mathrm{~N}(\mathrm{Qv}, \mathrm{STv}, \mathrm{t})>0$. Then from (ii) with $\alpha=1$ we have

$[1+\mathrm{aM}(\mathrm{ABu}, \mathrm{STv}, \mathrm{t})] * \mathrm{M}(\mathrm{Pu}, \mathrm{Qv}, \mathrm{t})$

$\geq \mathrm{a}[\mathrm{M}(\mathrm{ABu}, \mathrm{STv}, \mathrm{t}) * \mathrm{M}(\mathrm{Pu}, \mathrm{ABu}, \mathrm{t}) * \mathrm{M}(\mathrm{Qv}, \mathrm{STv}, \mathrm{t})$

* $\mathrm{M}(\mathrm{Pu}, \mathrm{STv}, \mathrm{t}) * \mathrm{M}(\mathrm{Qv}, \mathrm{ABu}, \mathrm{t})]+$

$\phi\left(\begin{array}{c}M(A B u, S T v, t), M(P u, A B u, t), M(Q v, S T v, t) \\ M(P u, S T v, t), M(Q v, A B u, t)\end{array}\right)$

$[1+\mathrm{aN}(\mathrm{ABu}, \mathrm{STv}, \mathrm{t})] \diamond \mathrm{N}(\mathrm{Pu}, \mathrm{Qv}, \mathrm{t})$

$\leq \mathrm{a}[\mathrm{N}(\mathrm{ABu}, \mathrm{STv}, \mathrm{t}) \diamond \mathrm{N}(\mathrm{Pu}, \mathrm{ABu}, \mathrm{t}) \diamond \mathrm{N}(\mathrm{Qv}, \mathrm{STv}, \mathrm{t}) \diamond$

$\mathrm{N}(\mathrm{Pu}, \mathrm{STv}, \mathrm{t}) \diamond \mathrm{N}(\mathrm{Qv}, \mathrm{ABu}, \mathrm{t})]+$

$\phi^{\prime}\left(\begin{array}{c}N(A B u, S T v, t), N(P u, A B u, t), N(Q v, S T v, t) \\ N(P u, S T v, t), N(Q v, A B u, t)\end{array}\right)$

$[1+\mathrm{a} 1] * \mathrm{M}(\mathrm{STv}, \mathrm{Qv}, \mathrm{t}) \geq \mathrm{a}[1 * 1 * \mathrm{M}(\mathrm{Qv}, \mathrm{STv}, \mathrm{t}) * 1 *$

$\mathrm{M}(\mathrm{Qv}, \mathrm{STv}, \mathrm{t})]+$

$$
\phi(1,1, M(Q v, S T v, t), 1, M(Q v, S T v, t))
$$

$[1+\mathrm{a} 1] \diamond \mathrm{N}(\mathrm{STv}, \mathrm{Qv}, \mathrm{t}) \leq \mathrm{a}\left[0 \diamond 0 \diamond \mathrm{N}(\mathrm{Qv}, \mathrm{STv}, \mathrm{t}) \diamond_{0} \diamond\right.$

$\mathrm{N}(\mathrm{Qv}, \mathrm{STv}, \mathrm{t})]+$

$$
\phi^{\prime}(0,0, N(Q v, S T v, t), 0, N(Q v, S T v, t)) .
$$

$\mathrm{M}(\mathrm{STv}, \mathrm{Qv}, \mathrm{t}) \geq \phi(1,1, \mathrm{M}(\mathrm{Qv}, \mathrm{STv}, \mathrm{t}), 1, \mathrm{M}(\mathrm{Qv}, \mathrm{STv}$, $\mathrm{t}))>\mathrm{M}(\mathrm{Qv}, \mathrm{STv}, \mathrm{t})$

$\mathrm{N}(\mathrm{STv}, \mathrm{Qv}, \mathrm{t}) \leq$

$\phi^{\prime}(0,0, N(Q v, S T v, t), 0, N(Q v, S T v, t)<\mathrm{N}(\mathrm{Qv}, \mathrm{ST}$,

t).
It is a contradiction. Hence Qv $=\mathrm{STv}$.....(xi)

Thus $\mathrm{ABu}=\mathrm{Pu}=\mathrm{STv}=\mathrm{Qv}=\mathrm{p}, \mathrm{say} \ldots \ldots .(\mathrm{xii})$

Since the pair $(\mathrm{P}, \mathrm{AB})$ is weakly compatible we have

$\mathrm{Pp}=\mathrm{PPu}=\mathrm{PABu}=\mathrm{ABPu}=\mathrm{ABABu}=\mathrm{ABp} \ldots . . .($ xiii $)$.

Suppose $r=M(p, P p, t)<1, r=N(p, P p, t)>0$. From (ii) with $\alpha=1, \mathrm{x}=\mathrm{p}, \mathrm{y}=\mathrm{v}$ we have

$[1+\mathrm{aM}(\mathrm{ABp}, \mathrm{p}, \mathrm{t})] * \mathrm{M}(\mathrm{Pp}, \mathrm{p}, \mathrm{t})$

$\geq \mathrm{a}[\mathrm{M}(\mathrm{ABp}, \mathrm{p}, \mathrm{t}) * 1 * 1 * \mathrm{M}(\mathrm{Pp}, \mathrm{p}, \mathrm{t}) * \mathrm{M}(\mathrm{p}, \mathrm{ABp}, \mathrm{t})]+$

$\phi(M(A B p, p, t), 1,1, M(P p, p, t), M(p, A B p, t))$,

$[1+\mathrm{aN}(\mathrm{ABp}, \mathrm{p}, \mathrm{t})] \diamond \mathrm{N}(\mathrm{Pp}, \mathrm{p}, \mathrm{t})$

$\leq \mathrm{a}\left[\mathrm{N}(\mathrm{ABp}, \mathrm{p}, \mathrm{t}) \diamond_{0} \diamond 0 \diamond \mathrm{N}(\mathrm{Pp}, \mathrm{p}, \mathrm{t}) \diamond \mathrm{N}(\mathrm{p}, \mathrm{ABp}, \mathrm{t})\right]+$

$\phi^{\prime}(\mathrm{N}(\mathrm{ABp}, \mathrm{p}, \mathrm{t}), 0,0, \mathrm{~N}(\mathrm{Pp}, \mathrm{p}, \mathrm{t}), \mathrm{N}(\mathrm{p}, \mathrm{ABp}, \mathrm{t}))$.

$\mathrm{M}(\mathrm{Pp}, \mathrm{p}, \mathrm{t}) \geq \varphi(\mathrm{r}, \mathrm{r}, \mathrm{r}, \mathrm{r}, \mathrm{r})>\mathrm{r}$ and

$\mathrm{N}(\mathrm{Pp}, \mathrm{p}, \mathrm{t}) \leq \phi^{\prime}(\mathrm{r}, \mathrm{r}, \mathrm{r}, \mathrm{r}, \mathrm{r})<\mathrm{r}$

It is a contradiction.

Hence $\mathrm{Pp}=\mathrm{p}$.Thus $\mathbf{A B p}=\mathbf{P} \mathbf{p}=\mathbf{p}$....(xiv)

Since the pair $(\mathrm{Q}, \mathrm{ST})$ is weakly compatible we have $\mathrm{Qp}=$ STp. Using (ii) with

$\alpha=1, x=u, y=p$ we can show that $Q p=p$.

Thus STp $=p=$ Qp....(xv).

Suppose $\mathrm{Bp} \neq \mathrm{p}$ and $\mathrm{r}=\mathrm{M}(\mathrm{p}, \mathrm{Bp}, \mathrm{t})<1, \mathrm{r}=\mathrm{N}(\mathrm{p}, \mathrm{Bp}, \mathrm{t})>0$.

From (ii) with $\mathrm{x}=\mathrm{Bp}, \mathrm{y}=\mathrm{p}, \alpha=1$ we have,

$[1+\mathrm{aM}(\mathrm{ABBp}, \mathrm{STp}, \mathrm{t})] * \mathrm{M}(\mathrm{PBp}, \mathrm{Qp}, \mathrm{t})$

$\geq \mathrm{a}[\mathrm{M}(\mathrm{ABB} p, \mathrm{STp}, \mathrm{t}) * \mathrm{M}(\mathrm{PBp}, \mathrm{ABBp}, \mathrm{t}) * \mathrm{M}(\mathrm{Qp}, \mathrm{STp}, \mathrm{t})$

* M(PBp,STp,t)* M(Qp, ABBp, t)]+

$\phi\left(\begin{array}{c}M(A B B p, S T p, t), M(P B p, A B B p, t), M(Q p, S T p, t) \\ M(P B p, S T p, t), M(Q p, A B B p, t)\end{array}\right)$

$1+\mathrm{aN}(\mathrm{ABBp}, \mathrm{STp}, \mathrm{t})] \diamond \mathrm{N}(\mathrm{PBp}, \mathrm{Qp}, \mathrm{t})$

$\leq \mathrm{a}[\mathrm{N}(\mathrm{ABB}, \mathrm{STp}, \mathrm{t}) \diamond \mathrm{N}(\mathrm{PBp}, \mathrm{ABBp}, \mathrm{t}) \diamond \mathrm{N}(\mathrm{Qp}, \mathrm{STp}, \mathrm{t})$

$\diamond \mathrm{N}(\mathrm{PBp}, \mathrm{STp}, \mathrm{t}) \diamond \mathrm{N}(\mathrm{Qp}, \mathrm{ABBp}, \mathrm{t})]+$ 
$\phi^{\prime}\left(\begin{array}{c}N(A B B p, S T p, t), N(P B p, A B B p, t), N(Q p, S T p, t) \\ N(P B p, S T p, t), N(Q p, A B B p, t)\end{array}\right)$

Since $\mathrm{AB}=\mathrm{BA}, \mathrm{PB}=\mathrm{BP}$, we have

$\mathrm{P}(\mathrm{Bp})=\mathrm{B}(\mathrm{Pp})=\mathrm{Bp}$ and $\mathrm{AB}(\mathrm{Bp})=\mathrm{B}(\mathrm{ABp})=\mathrm{Bp}$,

$[1+\mathrm{aM}(\mathrm{Bp}, \mathrm{STp}, \mathrm{t})] * \mathrm{M}(\mathrm{Bp}, \mathrm{Qp}, \mathrm{t})$

$\geq \mathrm{a}[\mathrm{M}(\mathrm{Bp}, \mathrm{STp}, \mathrm{t}) * \mathrm{M}(\mathrm{Bp}, \mathrm{Bp}, \mathrm{t}) * \mathrm{M}(\mathrm{Qp}, \mathrm{STp}, \mathrm{t})$

* $\mathrm{M}(\mathrm{Bp}, \mathrm{STp}, \mathrm{t}) * \mathrm{M}(\mathrm{Qp}, \mathrm{Bp}, \mathrm{t})]+$

$\phi\left(\begin{array}{c}M(B p, S T p, t), M(B p, B p, t), M(Q p, S T p, t) \\ M(B p, S T p, t), M(Q p, B p, t)\end{array}\right)$

$1+\mathrm{aN}(\mathrm{Bp}, \mathrm{STp}, \mathrm{t})] \diamond \mathrm{N}(\mathrm{Bp}, \mathrm{Qp}, \mathrm{t})$

$\leq \mathrm{a}[\mathrm{N}(\mathrm{Bp}, \mathrm{STp}, \mathrm{t}) \diamond \mathrm{N}(\mathrm{Bp}, \mathrm{Bp}, \mathrm{t}) \diamond \mathrm{N}(\mathrm{Qp}, \mathrm{STp}, \mathrm{t})$

$\diamond \mathrm{N}(\mathrm{Bp}, \mathrm{STp}, \mathrm{t}) \oslash \mathrm{N}(\mathrm{Qp}, \mathrm{Bp}, \mathrm{t})]+$

$\phi^{\prime}\left(\begin{array}{c}N(B p, S T p, t), N(B p, B p, t), N(Q p, S T p, t) \\ N(B p, S T p, t), N(Q p, B p, t)\end{array}\right)$.

$\mathrm{M}(\mathrm{Bp}, \mathrm{p}, \mathrm{t}) \geq \mathrm{a}[\mathrm{M}(\mathrm{Bp}, \mathrm{Bp}, \mathrm{t}) * \mathrm{M}(\mathrm{p}, \mathrm{p}, \mathrm{t}) * \mathrm{M}(\mathrm{Bp}, \mathrm{p}, \mathrm{t})$

$* \mathrm{M}(\mathrm{p}, \mathrm{Bp}, \mathrm{t})]+$

$\phi\left(\begin{array}{c}M(B p, p, t), M(B p, B p, t), M(p, p, t) \\ M(B p, p, t), M(p, B p, t)\end{array}\right)$,

$\mathrm{N}(\mathrm{Bp}, \mathrm{p}, \mathrm{t}) \leq \mathrm{a}[\mathrm{N}(\mathrm{Bp}, \mathrm{p}, \mathrm{t}) \diamond \mathrm{N}(\mathrm{Bp}, \mathrm{Bp}, \mathrm{t})$

$\diamond \mathrm{N}(\mathrm{p}, \mathrm{p}, \mathrm{t}) \diamond \mathrm{N}(\mathrm{Bp}, \mathrm{p}, \mathrm{t}) \diamond \mathrm{N}(\mathrm{p}, \mathrm{Bp}, \mathrm{t})]+$

$\phi^{\prime}\left(\begin{array}{c}N(B p, p, t), N(B p, B p, t), N(p, p, t) \\ N(B p, p, t), N(p, B p, t)\end{array}\right)$.

$\mathrm{M}(\mathrm{Bp}, \mathrm{p}, \mathrm{t}) \geq \phi(r, r, r, r, r)>\mathrm{r}, \mathrm{N}(\mathrm{Bp}, \mathrm{p}, \mathrm{t}) \leq \phi^{\prime}(\mathrm{r}, \mathrm{r}, \mathrm{r}, \mathrm{r}$, r) $<$ r.

It is a contradiction. Hence $\mathrm{Bp}=\mathrm{p}$.

Since $\mathrm{p}=\mathrm{ABp}$, we have $\mathbf{p}=\mathbf{A p}=\mathbf{B} \mathbf{p}=\mathbf{P p}$

Finally, we show that $\mathrm{Tp}=\mathrm{p}$ by putting $\mathrm{x}=\mathrm{p}$ and $\mathrm{y}=\mathrm{Tp}, \alpha$ $=1$ we have,

in (v), Suppose $\mathrm{Tp} \neq \mathrm{p}$ and $\mathrm{r}=\mathrm{M}(\mathrm{p}, \mathrm{Tp}, \mathrm{t})<1, \mathrm{r}=\mathrm{N}(\mathrm{p}, \mathrm{Tp}$, t) $>1$.
If $\mathrm{Tp} \neq \mathrm{p}$, then

$[1+\mathrm{aM}(\mathrm{ABp}, \mathrm{STTp}, \mathrm{t})] * \mathrm{M}(\mathrm{Pp}, \mathrm{QTp}, \mathrm{t})$

$\geq \mathrm{a}[\mathrm{M}(\mathrm{ABp}, \mathrm{STTp}, \mathrm{t}) * \mathrm{M}(\mathrm{Pp}, \mathrm{ABp}, \mathrm{t}) * \mathrm{M}(\mathrm{QTp}, \mathrm{STTp}, \mathrm{t})$

* M(Pp,STTp,t $) * \mathrm{M}(\mathrm{QTp}, \mathrm{ABp}, \mathrm{t})]+$

$\phi\left(\begin{array}{c}M(A B p, S T T p, t), M(P p, A B p, t), M(Q T p, S T T p, t) \\ M(P p, S T T p, t), M(Q T p, A B p, t)\end{array}\right)$

$1+\mathrm{aN}(\mathrm{ABp}, \mathrm{STTp}, \mathrm{t})] \diamond \mathrm{N}(\mathrm{Pp}, \mathrm{QTp}, \mathrm{t})$

$\leq \mathrm{a}[\mathrm{N}(\mathrm{ABp}, \mathrm{STTp}, \mathrm{t}) \diamond \mathrm{N}(\mathrm{Pp}, \mathrm{ABp}, \mathrm{t}) \diamond \mathrm{N}(\mathrm{QT}$, STTp, t $)$

$\diamond \mathrm{N}(\mathrm{Pp}, \mathrm{STTp}, \mathrm{t}) \diamond \mathrm{N}(\mathrm{QTp}, \mathrm{ABp}, \mathrm{t})]+$

$\phi^{\prime}\left(\begin{array}{c}N(A B p, S T T p, t), N(P p, A B p, t), N(Q T p, S T T p, t) \\ N(P p, S T T p, t), N(Q T p, A B p, t)\end{array}\right)$

Since $\mathrm{ST}=\mathrm{TS}, \mathrm{TQ}=\mathrm{QT}$, we have

$\mathrm{Q}(\mathrm{Tp})=\mathrm{T}(\mathrm{Qp})=\mathrm{Tp}$ and $\mathrm{ST}(\mathrm{Tp})=\mathrm{T}(\mathrm{STp})=\mathrm{Tp}$,

$[1+\mathrm{aM}(\mathrm{ABp}, \mathrm{Tp}, \mathrm{t})] * \mathrm{M}(\mathrm{Pp}, \mathrm{Tp}, \mathrm{t})$

$\geq \mathrm{a}[\mathrm{M}(\mathrm{ABp}, \mathrm{Tp}, \mathrm{t}) * \mathrm{M}(\mathrm{Pp}, \mathrm{ABp}, \mathrm{t}) * \mathrm{M}(\mathrm{Tp}, \mathrm{Tp}, \mathrm{t})$

$* \mathrm{M}(\mathrm{Pp}, \mathrm{Tp}, \mathrm{t}) * \mathrm{M}(\mathrm{Tp}, \mathrm{ABp}, \mathrm{t})]+$

$\phi\left(\begin{array}{c}M(A B p, T p, t), M(P p, A B p, t), M(T p, T p, t) \\ M(P p, T p, t), M(T p, A B p, t)\end{array}\right)$

$1+\mathrm{aN}(\mathrm{ABp}, \mathrm{Tp}, \mathrm{t})] \diamond \mathrm{N}(\mathrm{Pp}, \mathrm{Tp}, \mathrm{t})$

$\leq \mathrm{a}[\mathrm{N}(\mathrm{ABp}, \mathrm{Tp}, \mathrm{t}) \diamond \mathrm{N}(\mathrm{Pp}, \mathrm{ABp}, \mathrm{t}) \diamond \mathrm{N}(\mathrm{Tp}, \mathrm{Tp}, \mathrm{t})$

$\diamond \mathrm{N}(\mathrm{Pp}, \mathrm{Tp}, \mathrm{t}) \diamond \mathrm{N}(\mathrm{Tp}, \mathrm{ABp}, \mathrm{t})]+$

$\phi^{\prime}\left(\begin{array}{c}N(A B p, T p, t), N(P p, A B p, t), N(T p, T p, t) \\ N(P p, T p, t), N(T p, A B p, t)\end{array}\right)$.

$\mathrm{M}(\mathrm{p}, \mathrm{Tp}, \mathrm{t}) \geq \mathrm{a}[1 * 1 * \mathrm{M}(\mathrm{p}, \mathrm{Tp}, \mathrm{t}) * \mathrm{M}(\mathrm{Tp}, \mathrm{p}, \mathrm{t})]+$

$\phi\left(\begin{array}{c}M(p, T p, t), M(p, p, t), M(p, p, t) \\ M(p, T p, t), M(T p, p, t)\end{array}\right)$,

$\mathrm{N}(\mathrm{p}, \mathrm{Tp}, \mathrm{t}) \leq \mathrm{a}\left[00_{0} \diamond \mathrm{N}(\mathrm{p}, \mathrm{Tp}, \mathrm{t}) \diamond \mathrm{N}(\mathrm{Tp}, \mathrm{p}, \mathrm{t})+\right.$ 
$\phi^{\prime}\left(\begin{array}{c}N(p, T p, t), N(p, p, t), N(p, p, t) \\ N(p, T p, t), N(T p, p, t)\end{array}\right)$.

$\mathrm{M}(\mathrm{p}, \mathrm{Tp}, \mathrm{t}) \geq \phi(r, r, r, r, r)>\mathrm{r}$,

$\mathrm{N}(\mathrm{p}, \mathrm{Tp}, \mathrm{t}) \leq \phi^{\prime}(\mathrm{r}, \mathrm{r}, \mathrm{r}, \mathrm{r}, \mathrm{r})<\mathrm{r}$.

It is a contradiction. Hence $\mathrm{Tp}=\mathrm{p}$.

Since $\mathrm{p}=\mathrm{ST}$, we have $\mathrm{p}=\mathrm{Sp}$.

By combining the above results, $\mathrm{Ap}=\mathrm{Bp}=\mathrm{Sp}=\mathrm{Tp}=\mathrm{Pp}=$ $\mathrm{Qp}=\mathrm{p}$,

So $\mathrm{P}, \mathrm{Q}, \mathrm{A}, \mathrm{B}, \mathrm{S}$ and $\mathrm{T}$ have a fixed common fixed point $\mathrm{p}$.

\begin{abstract}
Now to prove uniqueness, if possible $\mathrm{p}_{0} \neq \mathrm{p}$ be another
\end{abstract} common fixed point $\mathrm{P}, \mathrm{Q}, \mathrm{A}, \mathrm{B}, \mathrm{S}$ and $\mathrm{T}$. Then

Suppose $\mathrm{p}_{0} \neq \mathrm{p}$ and $\mathrm{r}=\mathrm{M}\left(\mathrm{p}_{0}, \mathrm{p}, \mathrm{t}\right)<1, \mathrm{r}=\mathrm{N}\left(\mathrm{p}_{0}, \mathrm{p}, \mathrm{t}\right)>1$.

From (ii) with $\mathrm{x}=\mathrm{p}_{0}, \mathrm{y}=\mathrm{p}, \alpha=1$ we have,

$\left[1+\mathrm{aM}\left(\mathrm{ABp}_{0}, \mathrm{STp}, \mathrm{t}\right)\right] * \mathrm{M}\left(\mathrm{Pp}_{0}, \mathrm{Qp}, \mathrm{t}\right)$

$\geq \mathrm{a}\left[\mathrm{M}\left(\mathrm{ABp}_{0}, \mathrm{STp}, \mathrm{t}\right) * \mathrm{M}\left(\mathrm{Pp}_{0}, \mathrm{ABp}_{0}, \mathrm{t}\right) * \mathrm{M}(\mathrm{Qp}, \mathrm{STp}, \mathrm{t})\right.$

$\left.* \mathrm{M}\left(\mathrm{Pp}_{0}, \mathrm{STp}, \mathrm{t}\right) * \mathrm{M}\left(\mathrm{Qp}, \mathrm{ABp} \mathrm{p}_{0}, \mathrm{t}\right)\right]+$

$\phi\left(\begin{array}{c}M\left(A B p_{0}, S T p, t\right), M\left(P p_{0}, A B p_{0}, t\right), M(Q p, S T p, t) \\ M\left(P p_{0}, S T p, t\right), M\left(Q p, A B p_{0}, t\right)\end{array}\right)$

$\left.1+\mathrm{aN}\left(\mathrm{ABp}_{0}, \mathrm{STp}, \mathrm{t}\right)\right] \diamond \mathrm{N}\left(\mathrm{Pp}_{0}, \mathrm{Qp}, \mathrm{t}\right)$

$\leq \mathrm{a}\left[\mathrm{N}\left(\mathrm{ABp}_{0}, \mathrm{STp}, \mathrm{t}\right) \diamond \mathrm{N}\left(\mathrm{Pp}_{0}, \mathrm{ABp}_{0}, \mathrm{t}\right) \diamond \mathrm{N}(\mathrm{Qp}, \mathrm{STp}, \mathrm{t})\right.$

$\left.\diamond \mathrm{N}\left(\mathrm{Pp}_{0}, \mathrm{STp}, \mathrm{t}\right) \diamond \mathrm{N}\left(\mathrm{Qp}, \mathrm{ABp}_{0}, \mathrm{t}\right)\right]+$

$\phi^{\prime}\left(\begin{array}{c}N\left(A B p_{0}, S T p, t\right), N\left(P p_{0}, A B p_{0}, t\right), N(Q p, S T p, t) \\ N\left(P p_{0}, S T p, t\right), N\left(Q p, A B p_{0}, t\right)\end{array}\right)$

$\mathrm{M}\left(\mathrm{p}_{0}, \mathrm{p}, \mathrm{t}\right) \geq \mathrm{a}\left[\mathrm{M}\left(\mathrm{p}_{0}, \mathrm{p}_{0}, \mathrm{t}\right) * \mathrm{M}(\mathrm{p}, \mathrm{p}, \mathrm{t}) * \mathrm{M}\left(\mathrm{p}_{0}, \mathrm{p}, \mathrm{t}\right) * \mathrm{M}\left(\mathrm{p}, \mathrm{p}_{0}, \mathrm{t}\right)\right]$

$\phi\left(\begin{array}{c}M\left(p_{0}, p, t\right), M\left(p_{0}, p_{0}, t\right), M(p, p, t) \\ M\left(p_{0}, p, t\right), M\left(p, p_{0}, t\right)\end{array}\right)$

$\mathrm{N}\left(\mathrm{p}_{0}, \mathrm{p}, \mathrm{t}\right) \leq \mathrm{a}\left[\mathrm{N}\left(\mathrm{p}_{0}, \mathrm{p}_{0}, \mathrm{t}\right) \diamond \mathrm{N}(\mathrm{p}, \mathrm{p}, \mathrm{t})\right.$

$\left.\diamond \mathrm{N}\left(\mathrm{p}_{0}, \mathrm{p}, \mathrm{t}\right) \diamond \mathrm{N}\left(\mathrm{p}, \mathrm{p}_{0}, \mathrm{t}\right)\right]+$
$\phi^{\prime}\left(\begin{array}{c}N\left(p_{0}, p, t\right), N\left(p_{0}, p_{0}, t\right), N(p, p, t) \\ N\left(p_{0}, p, t\right), N\left(p, p_{0}, t\right)\end{array}\right)$.

$\mathrm{M}\left(\mathrm{p}_{0}, \mathrm{p}, \mathrm{t}\right) \geq \phi(r, r, r, r, r)>\mathrm{r}$,

$\mathrm{N}\left(\mathrm{p}_{0}, \mathrm{p}, \mathrm{t}\right) \leq \phi^{\prime}(\mathrm{r}, \mathrm{r}, \mathrm{r}, \mathrm{r}, \mathrm{r})<\mathrm{r}$.

It is a contradiction. Hence $\mathrm{p}_{0}=\mathrm{p}$.

Hence $\mathrm{p}$ is a common fixed point of $\mathrm{P}, \mathrm{Q}, \mathrm{A}, \mathrm{B}, \mathrm{S}$ and $\mathrm{T}$.

If we put $\mathrm{B}=\mathrm{T}=\mathrm{I}$ in theorem (3.1), we have the following:

Corollary 3.2. Let $\mathrm{P}, \mathrm{Q}, \mathrm{A}$ and $\mathrm{S}$ be self-mappings on $(\mathrm{X}, \mathrm{M}, \mathrm{N}, *, \diamond)$ such that

(i) $\mathrm{P}(\mathrm{X}) \subset \mathrm{S}(\mathrm{X})$ and $\mathrm{Q}(\mathrm{X}) \subset \mathrm{A}(\mathrm{X})$,

(ii) $\mathrm{P}$ and $\mathrm{A}$ are continuous or $\mathrm{Q}$ and $\mathrm{S}$ are continuous,

(iv) the pairs $(\mathrm{P}, \mathrm{A})$ and $(\mathrm{Q}, \mathrm{S})$ are weakly compatible,

(v) $[1+\mathrm{aM}(\mathrm{Ax}, \mathrm{Sy}, \mathrm{t})] * \mathrm{M}(\mathrm{Px}, \mathrm{Qy}, \mathrm{t})$

$\geq \mathrm{a}[\mathrm{M}(\mathrm{Ax}, \mathrm{Sy}, \mathrm{t}) * \mathrm{M}(\mathrm{Px}, \mathrm{Ax}, \mathrm{t}) * \mathrm{M}(\mathrm{Qy}, \mathrm{Sy}, \mathrm{t})$

* $\mathrm{M}(\mathrm{Px}, \mathrm{Sy}, \alpha \mathrm{t}) * \mathrm{M}(\mathrm{Qy}, \mathrm{Ax},(2-\alpha) \mathrm{t})]+$

$\phi\left(\begin{array}{c}M(A x, S y, t), M(P x, A x, t), M(Q y, S y, t) \\ M(P x, S y, \alpha t), M(Q y, A x,(2-\alpha) t)\end{array}\right)$

$[1+\mathrm{aN}(\mathrm{Ax}, \mathrm{Sy}, \mathrm{t})] \diamond \mathrm{N}(\mathrm{Px}, \mathrm{Qy}, \mathrm{t})$

$\leq \mathrm{a}[\mathrm{N}(\mathrm{Ax}, \mathrm{Sy}, \mathrm{t}) \diamond \mathrm{N}(\mathrm{Px}, \mathrm{Ax}, \mathrm{t}) \diamond \mathrm{N}(\mathrm{Qy}, \mathrm{Sy}, \mathrm{t})$

$\diamond \mathrm{N}(\mathrm{Px}, \mathrm{Sy}, \alpha \mathrm{t}) \diamond \mathrm{N}(\mathrm{Qy}, \mathrm{Ax},(2-\alpha) \mathrm{t})]+$

$\phi^{\prime}\left(\begin{array}{c}N(A x, S y, t), N(P x, A x, t), N(Q y, S y, t) \\ N(P x, S y, \alpha t), N(Q y, A x,(2-\alpha) t)\end{array}\right)$.

for every $\mathrm{x}, \mathrm{y} \in \mathrm{X}$, for all $\mathrm{t}>0$ and for every $\alpha \in(0,2)$, where $\varphi, \phi^{\prime} \in \Phi$ and $\mathrm{a} \in \mathbf{R}$,

Then $\mathrm{P}, \mathrm{Q}, \mathrm{A}$ and $\mathrm{S}$ have a unique common fixed point $\mathrm{p}$ in $\mathrm{X}$. If we put $\mathrm{P}=\mathrm{Q}=\mathrm{f}$ and $\mathrm{A}=\mathrm{S}=\mathrm{g}$ and $\mathrm{B}=\mathrm{T}=\mathrm{I}$ in theorem (3.1), we have the following:

Corollary 3.3. Let $\mathrm{f}$, g be self-mappings on (X,M,N, *, $\diamond)$ such that

(i) $\mathrm{f}(\mathrm{X}) \subset \mathrm{g}(\mathrm{X})$,

(ii) $\mathrm{g}(\mathrm{X})$ is complete,

(iv) the pair (f, g) is weakly compatible, 
(v) $[1+\mathrm{aM}(\mathrm{gx}, \mathrm{gy}, \mathrm{t})] * \mathrm{M}(\mathrm{fx}, \mathrm{fy}, \mathrm{t})$

$\geq \mathrm{a}[\mathrm{M}(\mathrm{gx}, \mathrm{gy}, \mathrm{t}) * \mathrm{M}(\mathrm{fx}, \mathrm{gx}, \mathrm{t}) * \mathrm{M}(\mathrm{fy}, \mathrm{gy}, \mathrm{t})$

* M(fx,gy, $\alpha \mathrm{t}) * \mathrm{M}(\mathrm{fy}, \mathrm{gx},(2-\alpha) \mathrm{t})]+$

$\phi\left(\begin{array}{c}M(g x, g y, t), M(f x, g x, t), M(f y, g y, t) \\ M(f x, g y, \alpha t), M(f y, g x,(2-\alpha) t)\end{array}\right)$

$[1+a N(g x, g y, t)] \diamond N(f x, f y, t)$

$\leq \mathrm{a}[\mathrm{N}(\mathrm{gx}$, gy, t $) \diamond \mathrm{N}(\mathrm{fx}, \mathrm{gx}, \mathrm{t}) \diamond \mathrm{N}(\mathrm{fy}, \mathrm{gy}, \mathrm{t})$

$\diamond \mathrm{N}(\mathrm{fx}, \mathrm{gy}, \alpha \mathrm{t}) \diamond \mathrm{N}(\mathrm{fy}, \mathrm{gx},(2-\alpha) \mathrm{t})]+$

$\phi^{\prime}\left(\begin{array}{c}N(g x, g y, t), N(f x, g x, t), N(f y, g y, t) \\ N(f x, g y, \alpha t), N(f y, g x,(2-\alpha) t)\end{array}\right)$.

for every $\mathrm{x}, \mathrm{y} \in \mathrm{X}$, for all $\mathrm{t}>0$ and for every $\alpha \in(0,2)$,

where $\varphi, \phi^{\prime} \in \Phi$ and $a \in \mathbf{R}$,

Then $\mathrm{f}, \mathrm{g}$ have a unique common fixed point $\mathrm{p}$ in $\mathrm{X}$.

\section{REFERENCES}

[1] Alaca, C., Turkoglu, D. and Yildiz, C., Fixed points in intuitionistic fuzzy metric spaces, Chaos, Solitons and Fractals, 29(2006), 1073- 1078.

[2] Atanassov, K., Intuitionistic fuzzy sets, Fuzzy sets and systems 20(1986) 87-96.

[3] Dubois, D. and Prade, H., Fuzzy Sets: Theory and Applications to Policy Analysis and Information Systems Plenum Press, New York, 1980.

[4] George, A. and Veeramani, P., On some results in fuzzy metric space, Fuzzy Sets and Systems, 64(1994), 395399.
[5] Grabiec, M. , Fixed points in fuzzy metric spaces, Fuzzy Sets and Systems 27 ( 1988), 385-389.

[6] Jungck, G. and Rhoades,B.E, Fixed point for set valued functions without continuity, Indian. J. Pure \& Applied Math.,29(3), 1998,227-238.

[7] Klement, E.P., Operations on fuzzy sets: an axiomatic approach, Inform. Sci. 27 (1984),221-232.

[8] Klement, E.P., Mesiar, R. and Pap, E., A characterization of the ordering of continuous t-norms, Fuzzy Sets and Systems 86 (1997), 189-195.

[9] Klement, E.P., Mesiar, R. and Pap, E., Triangular Norms, Kluwer Academic Publ., Trendsin Logic 8, Dordrecht 2000.

[10] Kramosil, O. and Michalek, J. , Fuzzy metric and statistical metric spaces, Kybernetica, 11 (1975), 326334.

[11] Menger, K., Statistical metrics, Proc. Nat. Acad. Sci. 28 (1942), 535-537.

[12] Park, J.H., Intuitionistic Fuzzy Metric Spaces, Chaos, Solitons and Fractals, 22(2004) 1039-1046.

[13] Rao, K.P.R., Rao, K.R.K. and Rao, T. Ranga, Common fixed point theorems in Sequentially Compact fuzzy metric spaces, International Mathematical Forum, 2(51)(2007)2543-2549.

[14] Sedghi, S., Common fixed point theorems for multivalued contractions, International Mathematical Forum, 2(31), (2007), 1499-1506.

[15] Sharma, S., Kutukcu, S., and Rathore, R.S., Common fixed point for multivalued mappings in intuitionistic fuzzy metric spaces, Korean Math.Soc.22(3)(2007),391399.

[16] Turkoglu, D., Alaca, C., Cho, Y.J. and Yildiz, C., Common fixed point theorems in intuitionistic fuzzy metric spaces, J. Appl. Math. And Computing, 22(12)(2006), 411-424.

[17] Zadeh, L. A., Fuzzy sets, Inform and Control 8 (1965), 338-353. 